\title{
Optimal Taxation with Endogenous Population Growth and the Risk of Environmental Disaster
}

\author{
Tapio Palokangas \\ University of Helsinki, Helsinki GSE, IIASA and IZA \\ P.O. Box 17, FIN-00014 University of Helsinki, Finland \\ Tel: +358503182263 Fax: +358919128736
}

\begin{abstract}
This study considers a market economy where firms produce goods from labor and capital and households supply labor, rear children, save in capital, promote their members' health and longevity by health care and derive utility from their consumption and children, without caring of their adult offspring. There is a risk that population growth and capital accumulation trigger a lethal environmental disaster. Optimal policy is solved by a game where the government is the leader and the representative household the follower. The solution yields precautionary taxes on both capital income and health care. Journal of Economic Literature: J13; O44; Q53; Q56; Q58

Keywords: random regime switch, stochastic dynamic programming, leader-follower game, environmental disaster, endogenous fertility, endogenous mortality
\end{abstract}

Email address: tapio.palokangas@helsinki.fi (Tapio Palokangas)

URL: http://blogs.helsinki.fi/palokang/ (Tapio Palokangas) 


\section{Introduction}

This document considers a market economy where firms produce goods from labor and capital and households save in capital, dictate the number of their children and spend on health care to improve their survival. The economy contains the externality that population growth and capital accumulation boost pollution that threatens to trigger a lethal environmental disaster. Could this externality be eliminated by (linear) taxation? This research question is examined by a dynamic game where the benevolent government is the leader and the representative household the follower.

For the sake of clarity, the disaster is taken as a random regime shift that occurs only once, with the post-event regime holding indefinitely. As pointed out by de Zeeuw and Zemel (2012), this restriction is not essential and models of recurrent events, where several shifts occur at random times with independent intervals, can be analyzed using the same methodology. Because the construction of different mortality rates for different cohorts would excessively complicate the analysis, then, following Becker (1981), it is assumed that the whole population has a uniform mortality rate, for simplicity.

Polasky et al. (2011) analyze how the threat of future regime shift affects the optimal management of natural resources. They focus on harvesting a renewable resource (e.g., fishery), whose growth rate is dependent on the regime and whose stock can trigger a regime shift. They show that the possibility of the regime shift makes the central planner precautionary, i.e., willing to maintain a larger stock of the resource. In this document, the government faces the risk of disaster due to pollution and behaves in precautionary manner by keeping the damaging stock (pollution) at a lower level.

Many dynamic models of pollution control assume smooth convex damage functions (e.g., van der Ploeg and de Zeeuw 1992, and Dockner and Long 1993), which ignores the effect of a potential regime shift on the optimal policy. Then, there is no need for precautionary measures against pollution: the policy maker should respond at the moment pollution occurs, but not beforehand. To examine the need for precautionary environmental policy, de Zeeuw and Zemel (2012) consider the management of a system that is subject to the risk of an abrupt and random jump in pollution damage. This document applies the same idea for the management of a market economy 
when pollution-related mortality is the damage.

Haurie and Moresino (2006), Polasky et al. (2011) and de Zeeuw and Zemel (2012) consider only the central planner that can fully control all resources of the economy. In contrast, this document examines the government in a market economy where microeconomic agents (households and firms) determine production, fertility and capital accumulation, unintentionally generating lethal emissions, but the government can use only linear taxes. Public policy is then constructed as a dynamic Stackelberg game where the government is the leader. This approach has the benefit that the suggested policy rules can be presented directly in terms of observable variables (e.g. prices and the interest rate).

Tsur and Zemel $(2008,2009)$ ignore population growth, but examine the possibility of climate change in a market economy where firms employ labor, capital and two energy inputs that are perfect substitutes: clean input that does not emit, and dirty input the emissions of which accumulate "hazardous" stock that threatens to trigger the climate change. As a result, they obtain a Pigouvian tax on the "hazardous" input. In contrast, this document considers endogenous population growth that may trigger the catastrophe. In order to avoid excessive complications in the model, the choice of energy inputs is ignored and it is assumed that population growth and capital accumulation generate "hazardous" pollution as a by-product.

Harford $(1997,1998)$ addresses the issue of environmental and population externality in a dynamic model where the individuals are altruistic toward their descendants and environmental pollution is a joint product of output. In his model, the social planner optimizes the utility of the representative individual. By comparing this optimum with the individuals' decisions, he shows that Pareto optimality requires both a pollution tax and a parental tax per child, because the former does not limit fertility enough to keep population stationary. To contribute to the discussion on this matter, this study adds Harford's parental tax into the potential tools of the government.

Palokangas (2018) and Lehmijoki and Palokangas (2019) examine optimal taxation in an economy where households dictate fertility and save for capital, while firms produce output from labor and capital, and population growth and capital accumulation generate pollution. In those studies, how- 
ever, there is no precautionary motive, for the current damage (mortality) is a smooth function of current pollution. In this document, in contrast, there is a precautionary motive: pollution triggers the damage (i.e., lethal environmental disaster) randomly at any moment of time.

The remainder of this document is organized as follows. Section 2 presents the basic structure of the economy, including the behavior of competitive firms. Then, a stochastic Stackelberg game is defined, with the government as the leader and the representative micro-household as the follower. Section 3 considers the household's and section 4 the government's behavior. Section 5 presents optimal public policy and section 6 summarizes the results.

\section{The economy as a whole}

\subsection{Population and labor supply}

In the model, time $t$ is continuous. Population $L$ grows at the rate that is equal to the fertility rate $f$ minus the mortality rate $m$ :

$$
\frac{1}{L} \frac{d L}{d t}=f-m, L(0)=L_{0}
$$

The units are normalized so that one unit of labor is needed to rear one newborn. Then, labor devoted to child rearing is equal to total fertility $f L$, and the remainder of the population, $N$, works in production:

$$
N \doteq L-f L=(1-f) L \Leftrightarrow n \doteq N / L=1-f
$$

\subsection{The goods market}

In the economy, there is only one good. The depreciation of capital is included in the production function of that good, so that the accumulation

of capital $K$ is given by $\frac{d K}{d t}$. Because (private) capital is the only asset in the model, private saving is equal to the accumulation of capital, $\frac{d K}{d t}$. The output 
of the good, $Y$, is used in consumption $C$, health care $H$ and investment $\frac{d K}{d t}$ :

$$
Y=C+H+\frac{d K}{d t}
$$

It is convenient to define consumption output $Y, C$, health care $H$ and capital $K$ in proportion to population $L$ :

$$
y \doteq \frac{Y}{L}, \quad c \doteq \frac{C}{L}, \quad h \doteq \frac{H}{L}, \quad k \doteq \frac{K}{L}
$$

Noting (1), (3) and (4), investment per head in the economy is defined by

$$
s \doteq \frac{d k}{d t}=\frac{d}{d t}\left(\frac{K}{L}\right)=\frac{1}{L} \frac{d K}{d t}-\frac{d L}{d t} \frac{K}{L^{2}}=\frac{1}{L} \frac{d K}{d t}+(m-f) k
$$

Because it is convenient to define investment per head $s \doteq \frac{d k}{d t}$ as a control in dynamic programming, then, by (5), private saving $\frac{d K}{d t}$ is given by

$$
\frac{d K}{d t}=[s+(f-m) k] L
$$

\subsection{Firms}

The firms produce output $Y$ from capital $K$ and labor input $N$ [cf. (2)] according to neoclassical technology:

$$
\begin{aligned}
& Y=F(K, N), \quad F_{K} \doteq \frac{\partial F}{\partial K}>0, \quad F_{N} \doteq \frac{\partial F}{\partial N}>0, \quad F_{K K} \doteq \frac{\partial^{2} F}{\partial K^{2}}<0, \\
& F_{N N} \doteq \frac{\partial^{2} F}{\partial N^{2}}<0, \quad F_{K N} \doteq \frac{\partial^{2} F}{\partial K \partial N}>0, \quad F \text { linearly homogeneous. }
\end{aligned}
$$


Noting (2), (4) and (7), output per head, $y$, can be defined as a function of capital per head, $k$, and the fertility rate $f$ as follows:

$$
\begin{aligned}
& 1-f=n \doteq N / L, \quad Y / L=F(k, n)=F(k, 1-f) \doteq y(k, f), \\
& y_{k} \doteq \frac{\partial y}{\partial k}=F_{K}(k, n)>0, \quad y_{f} \doteq \frac{\partial y}{\partial f}=-F_{N}(k, n)<0 .
\end{aligned}
$$

The representative firm maximizes its profit $\Pi$ by capital input $K$ and labor input $N$ according to technology (7), given the wage $w$ and the interest rate $r$. With (4) and (8), this implies

$$
\Pi \doteq \max _{K, N}[F(K, N)-w N-r K]=L \max _{k, n}[F(k, n)-w n-r k]
$$

Because the production function $F$ is subject to constant returns to scale (i.e., linearly homogeneous), then, in equilibrium, the marginal products of capital and labor, $F_{K}$ and $F_{N}$, are equal to the interest rate $r$ and the wage $w$, respectively, and total profit $\Pi$ is equal to zero [cf. (8) and (9)]:

$$
\begin{aligned}
& y(k, f)=F(k, n)=F_{B} n+F_{K} k=w n+r k=(1-f) w+r k, \\
& r=F_{K}(k, n)=y_{k}, \quad w=F_{N}(k, n), \quad y_{f}=-F_{N}(k, n)=-w .
\end{aligned}
$$

\subsection{Externality}

It is assumed that aggregate capital $K$ and aggregate population $L$ pollute according to the geometric average $P=K^{\gamma} L^{1-\gamma}=k^{\gamma} L$, where $0<\gamma<1$ is a constant. Then, the change of pollution, $v \doteq \frac{d P}{d t}$, is obtained from (1) and (6) as follows:

$$
\begin{aligned}
& \frac{1}{P} \frac{d P}{d t}=\frac{d \ln P}{d t}=\gamma \frac{d \ln k}{d t}+\frac{d \ln L}{d t}=\gamma \frac{1}{k} \frac{d k}{d t}+\frac{1}{L} \frac{d L}{d t}=\gamma \frac{s}{k}+f-m \\
& \Leftrightarrow v \doteq \frac{d P}{d t}=\left(\gamma \frac{s}{k}+f-m\right) P .
\end{aligned}
$$


With result (11), population $L$ can be replaced by pollution $P$ as a predetermined state variable in the model.

The probability of the environmental disaster, $\pi$, is assumed to be an increasing function of pollution $P$. Then, the disaster can be considered as a random shock $q$ with mean $\pi(P)$ as follows:

$$
q=\left\{\begin{array}{l}
1 \quad \text { with probability } \pi(P), \\
0 \quad \text { with probalility } 1-\pi(P),
\end{array} \text { where } \pi^{\prime}>0\right.
$$

The externality in the economy is the following: the environmental shock $q$ increases every individual's mortality rate $m$ simultaneously, but each individual can decrease its personal mortality rate $m$ by spending on its personal health care $h$ with increasing marginal costs. This function is specified by

$$
m=\chi(\delta q-h), \quad \chi^{\prime}>0, \quad \frac{d^{2}(-m)}{d h^{2}}=\chi^{\prime \prime}>0,
$$

where the constant $\delta>0$ is the effect of the shock $q$ in terms of output per head ( $=$ in terms of health care per head, $h$ ) and

$$
\left|\frac{d m}{d h}\right|=-\frac{d m}{d h}=\chi^{\prime}
$$

the marginal efficiency of personal health care $h$ in decreasing the personal mortality rate $m$.

The household chooses its saving per head, $s=\frac{d k}{d t}$, fertility rate $f$ and heath care per head, $h$. Because of the one-to-one correspondence between $h$ and $m$ through the function (13), then health care $h$ can be replaced by the mortality rate $m$ as the household's control in the model, for convenience. Denoting the inverse function of $\chi$ by $z(m) \doteq \chi^{-1}(m)$ in (13) yields

$$
h=\delta q-z(m), \quad z^{\prime} \doteq \frac{1}{\chi^{\prime}(m)}>0, \quad z^{\prime \prime} \doteq-\frac{\chi^{\prime \prime}}{\left(\chi^{\prime}\right)^{2}}<0 .
$$


The factors affecting the mortality rate $m$ [cf. (13)] affect also the level of health, $\ell$, but in the opposite direction: the environmental shock $q$ worsens every individual's health simultaneously, but each individual can improve its personal health $\ell$ by its personal health care $h$. Because the definition of health $\ell$ as a separate function of $q$ and $h$ would excessively complicate the analysis, and because it is technically convenient to handle the mortality rate as the household's control in the model, health $\ell$ and the mortality rate $m$ are defined as negatively associated joint products of the same process: ${ }^{1}$

$$
\ell(m), \quad \ell^{\prime}<0, \quad \ell^{\prime \prime} \text { exists. }
$$

\subsection{Public policy}

The government sets a poll tax $a \in \Re$ per head, the tax $\tau \in(-\infty, 1)$ on capital income $r K$, the parental tax $x \in \Re$ on the number of children, $f L$, and the $\operatorname{tax} b \in(-1, \infty)$ on health care $H$. If a tax is negative, then it is a subsidy. Any set of linear taxes that support Pareto optimum in the model is equivalent to those taxes. The government's budget is [cf. (4)]:

$$
a L+x f L+\tau r K+b H=0 \Leftrightarrow a+x f+\tau r k+b h=0 .
$$

In the model, the setup of public policy is a Stackelberg game as follows. The representative household is the follower that determines its consumption per head, $c$, its spending on health care per head, $h$, and its fertility rate $f$, taking the taxes $(a, x, \tau, b)$ and the environmental shock $q$ as given. The benevolent government is the leader that maximizes the representative household's utility by the taxes $(a, x, \tau, b)$, observing the follower's behavior, the behavior of the firms, (10), the budget constraint of its own, (17), and the risk of the regime shift $(12,15)$ due to pollution (11). The follower's and leader's behavior are examined in sections 3 and 4 .

\footnotetext{
${ }^{1}$ The mortality rate $m$ is introduced as a factor of utility through health $\ell$ [cf. (18)] only to ensure that the functions (26) and (42) can be strictly concave with respect to the mortality rate $m$ for realistic values of consumption per head, $c$, capital per head, $k$, and the mortality rate $m$.
} 


\section{The household}

\subsection{Utility}

According to Becker (1981), an individual derives its utility $c(t) f(t)^{\alpha}$, where $\alpha>0$ is a constant, from its consumption $c(t)$ and the fertility rate in its household, $f(t)$, at each time $t$. This study extends that framework by introducing personal health $\ell$ as the third factor of individual utility. Consequently, noting (16), periodic utility $u$ is a function of consumption per head, $c$, the fertility rate $f$ and the mortality rate $m$ as follows:

$$
u(t)=c(t) f(t)^{\alpha} \ell(m(t)), \quad \alpha>0, \quad \ell^{\prime}<0, \quad \ell^{\prime \prime} \text { exists. }
$$

Let $\rho$ be the constant rate of time preference for a hypothetical individual who could live forever. When an individual faces the mortality rate $m$, its probability of dying in a short time $d t$ is equal to $m d t$. Then, the probability of its survival beyond the period $[\zeta, t]$ is given by $e^{m(\zeta-t)}$, and its expected periodic utility at time $t \geq \zeta$ is $e^{m(\zeta-t)} u(t)$. Consequently, noting (16), the representative member's utility for the whole period $t \in[\zeta, \infty)$ in the household is given by

$$
\int_{\zeta}^{\infty} u(t)^{\sigma} e^{(\rho+m)(\zeta-t)} d t \text { with }(18), \quad 0<\sigma<1
$$

where $\sigma$ is a parameter and $\rho+m$ the effective rate of time preference with mortality. The closer $\sigma$ is to one, the more eagerly the household transfers resources from present to future by saving.

\subsection{Saving}

Investment $\frac{d K}{d t}$ is equal to private saving:

$$
\frac{d K}{d t}=w N+r K-C-h L-[a+x f+\tau r k+b h] L,
$$

where $w$ is the wage, $r$ the interest rate, $N$ labor supply, $w N$ labor income, $r K$ capital income, $C$ consumption, $h$ spending on health care per head, $h L$ 
total spending on health care and $[a+x f+\tau r k+b h] L$ tax expenditures [cf. (17)]. By (4), (6), (8), (15) and (20), consumption per head, c, can be defined as a function of the household's controls $(s, f, m)$, capital per head, $k$, taxes $(a, x, \tau, b)$, the wage $w$, the interest rate $r$ and the shock $q$ as follows:

$$
\begin{aligned}
& s+(f-m) k=\frac{1}{L} \frac{d K}{d t}=\frac{w N+r K-C}{L}-(1+b) h-a-x f-\tau r k \\
& =(1-f) w+r k-c+(1+b)[z(m)-\delta q]-a-x f-\tau r k \Leftrightarrow \\
& c=\widetilde{c}(s, f, m, k, a, x, \tau, b, w, r, q) \doteq \\
& w+(m-f+r-\tau r) k-s+(1+b)[z(m)-\delta q]-(w+x) f-a .
\end{aligned}
$$

\subsection{Transformation from real into virtual time}

The mortality rate $m$ can be eliminated from the discount factor of the utility function (19) by Uzawa's (1968) transformation:

$$
\theta(t)=(\rho+m) t \text { with } d t=\frac{d \theta}{\rho+m}
$$

Because $\theta(\zeta)=(\rho+m) \zeta, \theta(\infty)=\infty$ and $\frac{d t}{d \theta}=\frac{1}{\rho+m}>0$ hold true, one can define $\theta(t)$ as an alternative time variable and set the variables in terms of it. Noting (10) and (22), the utility function (19) with (18) and the constraint $s=\frac{d k}{d t}$ can be transformed into virtual time $\theta$ as follows:

$$
\begin{aligned}
& \int_{\zeta}^{\infty} \frac{c(\theta)^{\sigma} f(\theta)^{\alpha \sigma} \ell(m(\theta))^{\sigma}}{\rho+m(\theta)} e^{\zeta-\theta} d \theta, \\
& \frac{d k}{d \theta}=\frac{s(\theta)}{\rho+m(\theta)}, \quad k(0)=k_{0} .
\end{aligned}
$$

\subsection{Optimal behavior}

The household maximizes its utility (23) by investment per head, s, the fertility rate $f$ and the mortality rate $m$ subject to its consumption per head, (21), and its accumulation of wealth per head, (24), given the wage $w$, the 
interest rate $r$, the environmental shock $q$ and the taxes $(a, x, \tau, b)$. This defines the value function at initial time $\zeta$ as

$$
\Phi(k, a, x, \tau, b, w, r, q, \zeta) \doteq \max _{(s, f, m) \text { s.t. }(21),(24)} \int_{\zeta}^{\infty} \frac{c(\theta)^{\sigma} f(\theta)^{\alpha \sigma} \ell(m(\theta))^{\sigma}}{\rho+m(\theta)} e^{\zeta-\theta} d \theta
$$

Following Dixit and Pindyck (1994), and noting $s=\frac{d k}{d t}[$ cf. (5)], the Bellman equation for the household's program (25) is constructed as follows:

$$
\begin{aligned}
& \Phi(k, a, x, \tau, b, w, r, q, \zeta)=\underset{(s, f, m) \text { s.t. }(21)}{\max } \Lambda(s, f, m, k, a, x, \tau, b, w, r, q, \zeta) \text { with } \\
& \Lambda(s, f, m, k, a, x, \tau, b, w, r, q, \zeta) \\
& \doteq \frac{c^{\sigma} f^{\alpha \sigma} \ell}{\rho+m}+\frac{\partial \Phi}{\partial k} \frac{d k}{d \theta}=\frac{1}{\rho+m}\left[c^{\sigma} f^{\alpha \sigma} \ell(m)^{\sigma}+\frac{\partial \Phi}{\partial k} s\right]
\end{aligned}
$$

The first-order conditions for maximizing the function (26) by the controls $(s, f, m)$ subject to $(21)$ are given by

$$
\begin{aligned}
\frac{\partial \Lambda}{\partial s}= & \frac{1}{\rho+m}\left(\sigma c^{\sigma-1} f^{\alpha \sigma} \ell^{\sigma} \frac{\partial \widetilde{c}}{\partial s}+\frac{\partial \Phi}{\partial k}\right)=\frac{1}{\rho+m}\left(\frac{\partial \Phi}{\partial k}-\sigma c^{\sigma-1} f^{\alpha \sigma} \ell^{\sigma}\right)=0 \\
\Leftrightarrow & \frac{\partial \Phi}{\partial k}=\sigma c^{\sigma-1} f^{\alpha \sigma} \ell^{\sigma}, \\
\frac{\partial \Lambda}{\partial f}= & \frac{1}{\rho+m}\left(\alpha \sigma c^{\sigma} f^{\alpha \sigma-1} \ell^{\sigma}+\sigma c^{\sigma-1} f^{\alpha \sigma} \ell^{\sigma} \frac{\partial \widetilde{c}}{\partial f}\right)=\sigma \frac{c^{\sigma-1} f^{\alpha \sigma} \ell^{\sigma}}{\rho+m}\left(\alpha \frac{c}{f}-\frac{\partial \widetilde{c}}{\partial f}\right) \\
= & \sigma \frac{c^{\sigma-1} f^{\alpha \sigma} \ell^{\sigma}}{\rho+m}\left(\alpha \frac{c}{f}-w-x-k\right)=0 \Leftrightarrow f=\frac{\alpha c}{w+k+x}, \\
\frac{\partial \Lambda}{\partial m}= & \frac{1}{\rho+m}\left[\sigma c^{\sigma-1} f^{\alpha \sigma} \ell^{\sigma} \frac{\partial \widetilde{c}}{\partial m}+\sigma c^{\sigma} f^{\alpha \sigma} \ell^{\sigma-1} \ell^{\prime}-\frac{\Lambda}{\rho+m}\right] \\
= & \frac{1}{\rho+m}\left\{\sigma c^{\sigma-1} f^{\alpha \sigma} \ell(m)^{\sigma}\left[k+(1+b) z^{\prime}(m)\right]+\sigma c^{\sigma} f^{\alpha \sigma} \ell(m)^{\sigma-1} \ell^{\prime}(m)\right. \\
& \left.-\frac{\Lambda}{\rho+m}\right\}=0 \Leftrightarrow k+(1+b) z^{\prime}=\underbrace{\frac{\Lambda /(\rho+m)}{\sigma c^{\sigma-1} f^{\alpha \sigma} \ell^{\sigma}}}_{+}-c \underbrace{\frac{\ell^{\prime}}{\ell}}_{-}>0 .
\end{aligned}
$$


When the mortality rate $m$ is held constant, the function $\Lambda$ is strictly concave in controls $s$ and $f$. To obtain a unique equilibrium for the household, the strict concavity of $\Lambda$ must be extended for all controls $(s, f, m)$. This is done by examining the second-order partial derivative of $\Lambda$ with respect to $m$, which is obtained by (15), (19), (21) and (29) as follows:

$$
\begin{aligned}
\frac{\partial^{2} \Lambda}{\partial m^{2}}=\underbrace{\frac{1}{\rho+m}}_{+} & \{(\underbrace{\sigma-1}_{-}) \underbrace{\sigma c^{\sigma-2} f^{\alpha \sigma} \ell^{\sigma}}_{+} \underbrace{\left[k+(1+b) z^{\prime}\right]^{2}}_{+}+\underbrace{\sigma c^{\sigma-1} f^{\alpha \sigma} \ell^{\sigma}(1+b)}_{+} \underbrace{z^{\prime \prime}}_{-} \\
& +\underbrace{\sigma^{2} c^{\sigma-1} f^{\alpha \sigma} \ell^{\sigma-1}}_{+}[\underbrace{k+(1+b) z^{\prime}}_{+} \underbrace{\ell^{\prime}}_{-}+(\underbrace{\sigma-1}_{-}) \underbrace{\sigma c^{\sigma} f^{\alpha \sigma} \ell^{\sigma-2}\left(\ell^{\prime}\right)^{2}}_{+} \\
& +\underbrace{\sigma c^{\sigma} f^{\alpha \sigma} \ell^{\sigma-1}}_{+} \ell^{\prime \prime}+\underbrace{\frac{\Lambda}{(\rho+m)^{2}}}_{+}\} .
\end{aligned}
$$

If, in this equation, the negative effects of the mortality rate $m$ dominate over the positive intertemporal effect of the effective discount rate $\rho+m$ and the ambiguous effect of the mortality rate $m$ through the second derivative $\ell^{\prime \prime}$, then $\frac{\partial^{2} \Lambda}{\partial m^{2}}<0$ holds true and the function $\Lambda$ is strictly concave. Furthermore, by (29), one obtains

$$
\frac{\partial^{2} \Lambda}{\partial m \partial b}=\frac{\sigma c^{\sigma-1} f^{\alpha \sigma} \ell^{\sigma}}{\rho+m} z^{\prime}>0
$$

Thus, differentiating equation (29) totally yields the mortality function

$$
m=M(k, w, r, q, a, x, \tau, b, \zeta) \text { with } \frac{\partial M}{\partial b}=-\frac{\partial^{2} \Lambda}{\partial m \partial b} / \frac{\partial^{2} \Lambda}{\partial m^{2}}>0
$$

Results (28) and (30) can be explained as follows. An increase in the parental tax per child, $x$, decreases incentives to rear children (i.e., the fertility rate $f$ falls relative to consumption $c$ ). When capital per head, $k$, increases, it is more difficult for the household to save that capital $k$ for each newborn. This as well decreases incentives to rear children (i.e. $f$ falls). An increase in the tax on health care, $b$, discourages health care, increasing the 
mortality rate $m$.

The solution of dynamic programming is based on finding a specification for the value function $\Phi$. Then, one can use Merton's (1971) rule as follows. In the steady state $s=0$, from the Bellman equation (26) it follows that

$$
\left(\arg \max _{(s, f, m) \text { s.t. }(21)} \Lambda\right)_{s=0}=\left(\arg \max _{(s, f, m) \text { s.t. }(21)} \frac{c^{\sigma} f^{\alpha \sigma} \ell^{\sigma}}{\rho+m}\right)_{s=0 .}
$$

Thus, one can try the simplest case where the value function $\Phi$ is a positive constant $\vartheta$ times the maximized periodic utility in virtual time:

$$
\Phi \doteq \vartheta \max _{(s, f, m) \text { s.t. }(21)} \frac{c^{\sigma} f^{\alpha \sigma} \ell^{\sigma}}{\rho+m} .
$$

Plugging (31) into the Bellman equation (26) in the steady state $s=0$ yields

$$
\vartheta=1
$$

Thus, the value function (31) becomes

$$
\Phi \doteq \max _{(s, f, m) \text { s.t. }(21)} \frac{c^{\sigma} f^{\alpha \sigma} \ell^{\sigma}}{\rho+m}
$$

Inserting this into the first-order condition (27) and noting (21) yield

$$
\begin{aligned}
& \sigma c^{\sigma-1} f^{\alpha \sigma} \ell^{\sigma}=\frac{\partial \Phi}{\partial k}=\sigma \frac{c^{\sigma-1} f^{\alpha \sigma} \ell^{\sigma}}{\rho+m} \frac{\partial \widetilde{c}}{\partial k} \Leftrightarrow \rho+m=\frac{\partial \widetilde{c}}{\partial k}=m-f+r-\tau r \\
& \Leftrightarrow f=(1-\tau) r-\rho .
\end{aligned}
$$

The household's response functions are the fertility rate relative to consumption, (28), and the fertility rate (34). The government takes these together with the firm's responses (10) as constraints in its optimization. 


\section{The government}

\subsection{Setup for public policy}

The definition of pollution, (11), determines the fertility rate $f$ as a function of the controls $(s, v, m)$ and state variables $(k, P)$ :

$f(s, v, m, k, P) \doteq m+\frac{v}{P}-\gamma \frac{s}{k}, \quad \frac{\partial f}{\partial s}=-\frac{\gamma}{k}<0, \quad \frac{\partial f}{\partial v}=\frac{1}{P}>0, \quad \frac{\partial f}{\partial m}=1$, $\frac{\partial f}{\partial k}=\gamma \frac{s}{k^{2}}, \quad \frac{\partial f}{\partial P}=-\frac{v}{P^{2}}$.

The government balances its budget (17) by the poll tax $a$. Because there is one-to-one correspondence from the other taxes $(\tau, x, b)$ to $(s, v, m)$ through the system (21) [with (34)], (35) [with (28)] and (30), investment per head, $s=\frac{d k}{d t}$, the change of pollution, $v=\frac{d P}{d t}$, and the mortality rate $m$ can replace the taxes $(\tau, x, b)$ as the government's controls in the model.

Inserting the production function (10), the government's budget (17) and the fertility function (35) into the household's consumption per head, (21), it is possible to define consumption per head, $c$, as a function of the government's controls $(s, v, m)$ and the state variables $(k, P)$ as follows:

$$
c=\widehat{c}(s, v, m, k, P, q)=\widetilde{c}=y(k, f)+(m-f) k-s-\delta q+z(m),
$$

with the partial derivatives

$$
\begin{aligned}
& \frac{\partial \widehat{c}}{\partial s}=\left(y_{f}-k\right) \frac{\partial f}{\partial s}-1, \quad \frac{\partial \widehat{c}}{\partial v}=\left(y_{f}-k\right) \frac{\partial f}{\partial v}=\frac{y_{f}-k}{P}, \\
& \frac{\partial \widehat{c}}{\partial m}=k+z^{\prime}+\left(y_{f}-k\right) \frac{\partial f}{\partial m}, \quad \frac{\partial \widehat{c}}{\partial q}=-\delta, \quad \frac{\partial \widehat{c}}{\partial P}=\left(y_{f}-k\right) \frac{\partial f}{\partial P}, \\
& \frac{\partial \widehat{c}}{\partial k}=y_{k}+m-f+\left(y_{f}-k\right) \frac{\partial f}{\partial k} .
\end{aligned}
$$

By (22), the constraint $v=\frac{d P}{d t}$ can be written in virtual time $\theta$ as follows:

$$
\frac{d P}{d \theta}=\frac{v(\theta)}{\rho+m(\theta)}, \quad P(0)=P_{0} .
$$




\subsection{Optimization}

The government maximizes the representative household's welfare (23) by its controls $(s, v, m)$ subject to the occurrence of the environmental shock, (12), the accumulation of capital per head and aggregate pollution, (24) and (38), and the determination of the fertility rate and consumption per head, (35) and (36). Thus, its value function at initial time $\zeta$ is defined by

$$
\Psi(k, P, q, \zeta) \doteq \max _{\substack{(s(\zeta), v(\zeta), m(\zeta)) \\ \text { s.t. }(24),(35),(36),(38)}} \int_{\zeta}^{\infty} \frac{c(\theta)^{\sigma} f(\theta)^{\alpha \sigma} \ell(m(\theta))^{\sigma}}{\rho+m(\theta)} e^{\zeta-\theta} d \theta
$$

where $q=0$ holds true before and $q=1$ after the shock. Noting (39), one can define the relative damage of the shock in terms of welfare as follows:

$$
D(k, P, \zeta) \doteq \frac{\Psi(k, P, 0, \zeta)-\Psi(k, P, 1, \zeta)}{\Psi(k, P, 0, \zeta)}
$$

The following result is proven in the Appendix:

Proposition 1. If the loss of income due to the shock, $\delta$, is small relative to consumption per head before the shock, $\left.c\right|_{q=0}$, (e.g., if $\frac{\delta}{\left.c\right|_{q=0}}$ is less than $10 \%)$, then the relative damage of the shock in terms of welfare, (40), is approximately in fixed proportion $\sigma$ to it, $D(k, P, \zeta) \approx \sigma \frac{\delta}{c_{q=0}}$.

The parameter $\sigma \in(0,1)$ tells, how willing the households are to save for future in capital, i.e., $\frac{1}{1-\sigma}$ is the elasticity of intertemporal substitution. [cf. (19)]. If $\sigma$ is close to zero, then relative damage of the shock is insignificant in terms of current consumption. The closer $\sigma$ is to one, the greater the relative damage $D$ is in terms of current consumption.

At the occurrence of the environmental shock, $q$ jumps permanently from 0 to 1 [cf. (12)], changing welfare (39) from $\Psi(k, P, 0, \zeta)$ into $\Psi(k, P, 1, \zeta)$. Thus, by Kamien and Schwartz (1981) and Dixit and Pindyck (1994), the Bellman equation for the government's program is [cf. (12) and (39)]

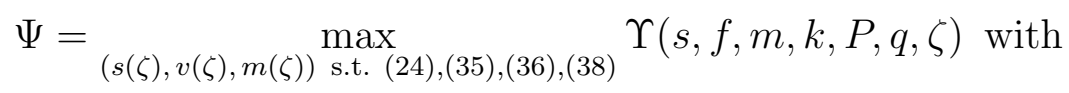




$$
\begin{aligned}
& \Upsilon(s, f, m, k, P, q, \zeta) \doteq \frac{c^{\sigma} f^{\alpha \sigma} \ell^{\sigma}}{\rho+m}+\frac{\partial \Psi}{\partial k}(k, P, q, \zeta) \frac{d k}{d \theta}+\frac{\partial \Psi}{\partial P}(k, P, q, \zeta) \frac{d P}{d \theta} \\
& +\pi(P)[\Psi(k, P, 1, \zeta)-\Psi(k, P, q, \zeta)] \\
& =\frac{1}{\rho+m}\left[c^{\sigma} f^{\sigma \alpha} \ell^{\sigma}+\frac{\partial \Psi}{\partial k}(k, P, 1, \zeta) s+\frac{\partial \Psi}{\partial P}(k, P, 1, \zeta) v\right] \\
& \quad+\pi(P)[\Psi(k, P, 1, \zeta)-\Psi(k, P, q, \zeta)],
\end{aligned}
$$

where the fertility rate $f$ and consumption per head, $c$, are determined by (35) and (36), $\pi(P)$ is the probability of the environmental shock [cf. (12)] and the difference $\Psi(k, P, 1, \zeta)-\Psi(k, P, q, \zeta)$ is the immediate change of welfare due to that shock. Note that the latter term $\pi[\Psi(k, P, 1, \zeta)-\Psi(k, P, q, \zeta)]$ vanishes entirely after the shock when $q=1$ holds true.

From the equilibrium condition $y_{f}=-w$ [cf. (10)] and the household's first-order condition (28) it follows that

$$
x=\alpha c / f-w-k=\alpha c / f+y_{f}-k .
$$

Noting (35), (37) and (43), the first-order conditions for the maximization (41) subject to (42) are obtained as follows:

$$
\begin{aligned}
0 & =\frac{\partial \Upsilon}{\partial s}=\frac{1}{\rho+m}\left[c^{\sigma} f^{\alpha \sigma} \ell^{\sigma}\left(\frac{\sigma}{c} \frac{\partial \widehat{c}}{\partial s}+\frac{\sigma \alpha}{f} \frac{\partial f}{\partial s}\right)+\frac{\partial \Psi}{\partial k}\right] \\
& =\frac{1}{\rho+m}\left\{\sigma c^{\sigma-1} f^{\alpha \sigma} \ell^{\sigma}\left[\left(y_{f}-k+\alpha \frac{c}{f}\right) \frac{\partial f}{\partial s}-1\right]+\frac{\partial \Psi}{\partial k}\right\} \\
& =\frac{1}{\rho+m}\left[\sigma c^{\sigma-1} f^{\alpha \sigma} \ell^{\sigma}\left(x \frac{\partial f}{\partial s}-1\right)+\frac{\partial \Psi}{\partial k}\right] \\
& =\frac{1}{\rho+m}\left[-\sigma c^{\sigma-1} f^{\alpha \sigma} \ell^{\sigma}\left(x \frac{\gamma}{k}+1\right)+\frac{\partial \Psi}{\partial k}\right], \\
0 & =\frac{\partial \Upsilon}{\partial v}=\frac{1}{\rho+m}\left[c^{\sigma} f^{\alpha \sigma} \ell^{\sigma}\left(\frac{\sigma}{c} \frac{\partial \widehat{c}}{\partial v}+\frac{\sigma \alpha}{f} \frac{\partial f}{\partial v}\right)+\frac{\partial \Psi}{\partial P}\right] \\
& =\frac{1}{\rho+m}\left[\sigma c^{\sigma-1} f^{\alpha \sigma} \ell^{\sigma}\left(y_{f}-k+\alpha \frac{c}{f}\right) \frac{\partial f}{\partial v}+\frac{\partial \Psi}{\partial P}\right]
\end{aligned}
$$




$$
\begin{aligned}
& =\frac{1}{\rho+m}\left(\sigma c^{\sigma-1} f^{\alpha \sigma} \ell^{\sigma} x \frac{\partial f}{\partial v}+\frac{\partial \Psi}{\partial P}\right)=\frac{1}{\rho+m}\left(\sigma c^{\sigma-1} f^{\alpha \sigma} \ell^{\sigma} \frac{x}{P}+\frac{\partial \Psi}{\partial P}\right), \\
0 & =\frac{\partial \Upsilon}{\partial m}=\frac{1}{\rho+m}\left[c^{\sigma} f^{\alpha \sigma} \ell^{\sigma}\left(\frac{\sigma}{c} \frac{\partial \widehat{c}}{\partial m}+\frac{\sigma \alpha}{f} \frac{\partial f}{\partial m}+\sigma \frac{\ell^{\prime}}{\ell}\right)-\frac{\Upsilon}{\rho+m}\right] \\
& =\frac{1}{\rho+m}\left\{\sigma c^{\sigma-1} f^{\alpha \sigma} \ell^{\sigma}\left[\left(y_{f}-k+\alpha \frac{c}{f}\right) \frac{\partial f}{\partial m}+k+z^{\prime}+c \frac{\ell^{\prime}}{\ell}\right]-\frac{\Upsilon}{\rho+m}\right\} \\
& =\frac{1}{\rho+m}\left[\sigma c^{\sigma-1} f^{\alpha \sigma} \ell^{\sigma}\left(x+k+z^{\prime}+c \frac{\ell^{\prime}}{\ell}\right)-\frac{\Upsilon}{\rho+m}\right] \Leftrightarrow \\
& \frac{1}{\sigma c^{\sigma-1} f^{\alpha \sigma} \ell^{\sigma}} \frac{\Upsilon}{\rho+m}-c \frac{\ell^{\prime}}{\ell}=x+k+z^{\prime} .
\end{aligned}
$$

The function $\Upsilon$ [cf. (42)] is strictly concave in $(s, v)$. To ensure that the government's equilibrium is unique, this property is extended by assuming that the function $\Upsilon$ as well is strictly concave in its arguments $(s, f, m)$.

\subsection{Solution}

In a steady state with $s=v=0$, from the Bellman equation (41) with (42) it follows that

$$
\left(\arg \max _{\substack{(s(\zeta), v(\zeta), m(\zeta)) \text { s.t. } \\(24),(35),(36),(38)}} \Upsilon\right)_{s=v=0}=\left(\arg \max _{\substack{(s(\zeta), v(\zeta), m(\zeta)) \text { s.t. } \\(24),(35),(36),(38)}} \frac{c^{\sigma} f^{\alpha \sigma} \ell^{\sigma}}{\rho+m}\right)_{s=v=0 \text {. }}
$$

Thus, one can try the simplest case where the value function $\Psi$ is a positive constant $\varpi$ times the maximized periodic utility in virtual time:

$$
\begin{aligned}
& \Psi(k, P, q, \zeta)=\varpi \max _{(s(\zeta), v(\zeta), m(\zeta)) \text { s.t. }(24),(35),(36),(38)} \frac{c^{\sigma} f^{\alpha \sigma} \ell^{\sigma}}{\rho+m}>0, \\
& \varpi>0, \quad \bar{\Psi}(k, P, \zeta) \doteq \Psi(k, P, 1, \zeta),
\end{aligned}
$$

where $\bar{\Psi}$ is the value after the disaster when $q=1$. Noting (35), (37), (43) and (47), the partial derivatives of the value function (47) with respect to 
the state variables $(k, P)$ are obtained as follows:

$$
\begin{aligned}
\frac{\partial \Psi}{\partial k} & =\Psi \frac{\partial \ln \Psi}{\partial k}=\Psi \frac{\sigma}{c} \frac{\partial \widehat{c}}{\partial k}+\frac{\sigma \alpha}{f} \frac{\partial f}{\partial k}=\Psi \frac{\sigma}{c}\left(\frac{\partial \widehat{c}}{\partial k}+\alpha \frac{c}{f} \frac{\partial f}{\partial k}\right) \\
& =\Psi \frac{\sigma}{c}\left[y_{k}+m-f+\left(y_{f}-k+\alpha \frac{c}{f}\right) \frac{\partial f}{\partial k}\right] \\
& =\Psi \frac{\sigma}{c}\left(y_{k}+m-f+x \frac{\partial f}{\partial k}\right)=\Psi \frac{\sigma}{c}\left(y_{k}+m-f+x \gamma \frac{s}{k^{2}}\right) \\
\frac{\partial \Psi}{\partial P} & =\Psi \frac{\partial \ln \Psi}{\partial P}=\Psi \frac{\sigma}{c}\left(\frac{\partial \widehat{c}}{\partial P}+\alpha \frac{c}{f} \frac{\partial f}{\partial P}\right)=\Psi \frac{\sigma}{c}\left(y_{f}-k+\alpha \frac{c}{f}\right) \frac{\partial f}{\partial P}=\Psi \frac{\sigma}{c} x \frac{\partial f}{\partial P} \\
& =-\Psi \frac{\sigma}{c} x \frac{v}{P^{2}} .
\end{aligned}
$$

Dividing the Bellman equation (41) and (42) by the value function (47) and noting the definition of the relative damage, (40), yield

$$
\begin{aligned}
1 & =\frac{\Upsilon}{\Psi}=\frac{c^{\sigma} f^{\alpha \sigma}}{\rho+m} \frac{1}{\Psi}+\frac{1}{\Psi} \frac{\partial \Psi}{\partial k} \frac{d k}{d \theta}+\frac{1}{\Psi} \frac{\partial \Psi}{\partial P} \frac{d P}{d \theta}+\pi(P) \frac{\Psi(k, P, 1, \zeta)-\Psi(k, P, q, \zeta)}{\Psi(k, P, q, \zeta)} \\
& = \begin{cases}\frac{1}{\varpi}+\frac{1}{\Psi} \frac{\partial \Psi}{\partial k} \frac{d k}{d \theta}+\frac{1}{\Psi} \frac{\partial \Psi}{\partial P} \frac{d P}{d \theta}-\pi(P) D(k, P, \zeta) & \text { for } q=0, \\
\frac{1}{\varpi}+\frac{1}{\Psi} \frac{\partial \Psi}{\partial k} \frac{d k}{d \theta}+\frac{1}{\Psi} \frac{\partial \Psi}{\partial P} \frac{d P}{d \theta} & \text { for } q=1 .\end{cases}
\end{aligned}
$$

In this study, the steady-state value of a variable is denoted by superscript $(*)$. There are different steady states before $(q=0)$ and after $(q=1)$ the shock. Because (50) holds in both of these steady states where $\frac{d P}{d \theta}=\frac{d k}{d \theta}=0$ hold true, the multiplier $\varpi$ is piecewise constant as follows:

$$
\left.\varpi\right|_{q=0}=\frac{1}{1+\pi^{*} D^{*}}<1,\left.\quad \varpi\right|_{q=1}=1,
$$

where $\pi^{*} \doteq \pi\left(\left.P^{*}\right|_{q=0}\right)$ is the probability of the disaster [cf. (12)], $D^{*} \doteq$ $D\left(\left.k^{*}\right|_{q=0},\left.P^{*}\right|_{q=0}, \xi\right)$ the relative damage [cf. (40)] and $\pi^{*} D^{*}$ the expected relative damage in the steady state before the occurrence of the shock. 


\section{Optimal policy}

\subsection{The parental tax per child}

It is assumed that the relative change of pollution, $\frac{v}{P}$, is either negative or positive, but small enough for $\frac{v}{P}<\frac{\rho+m}{\varpi}$. Inserting the value function (47) and its partial derivative (49) into the government's first-order condition (45) and noting the government's fertility function (35) yield

$$
\begin{aligned}
0 & =(\rho+m) \frac{\partial \Upsilon}{\partial v}=\sigma c^{\sigma-1} f^{\alpha \sigma} \ell^{\sigma} \frac{x}{P}+\frac{\partial \Psi}{\partial P}=\sigma c^{\sigma-1} f^{\alpha \sigma} \ell^{\sigma} \frac{x}{P}-\Psi \frac{\sigma}{c} x \frac{v}{P^{2}} \\
& =\frac{\Psi}{P} \frac{\sigma}{c} x\left(\frac{c^{\sigma} f^{\alpha \sigma} \ell^{\sigma}}{\Psi}-\frac{v}{P}\right)=\frac{\Psi}{P} \frac{\sigma}{c} x(\underbrace{\left.\frac{\rho+m}{\varpi}-\frac{v}{P}\right) \Leftrightarrow x=0 .}_{+}
\end{aligned}
$$

Thus, in contrast to Harford $(1997,1998)$, the parental tax per child is not positive in this case:

Proposition 2. The parental tax per child can be eschewed, $x=0$.

Because the other taxes eliminate the externality through pollution and mortality, this tax is unnecessary.

\subsection{Taxing capital income}

Plugging $x=0\left[\mathrm{cf}\right.$. (52)], the profit maximization condition $y_{k}=r[\mathrm{cf}$. (10)] and the value function (47) into the partial derivative (48) and the first-order condition (44), one obtains

$$
\begin{aligned}
0 & =\frac{\partial \Psi}{\partial k}-\sigma c^{\sigma-1} f^{\alpha \sigma} \ell^{\sigma}\left(x \frac{\gamma}{k}+1\right) \\
& =\Psi \frac{\sigma}{c}\left(y_{k}+m-f+x \gamma \frac{s}{k^{2}}\right)-\sigma c^{\sigma-1} f^{\alpha \sigma}\left(x \frac{\gamma}{k}+1\right) \\
& =\Psi \frac{\sigma}{c}(r+m-f)-\frac{\sigma}{c} \frac{\Psi}{\varpi}(\rho+m)=\Psi \frac{\sigma}{c}\left(r+m-f-\frac{\rho+m}{\varpi}\right) \\
& \Leftrightarrow r=f-m+\frac{\rho+m}{\varpi} .
\end{aligned}
$$


Because the ratio of the difference between the fertility and mortality rates to the sum of the rate of time preference and the mortality rate, $\frac{f-m}{\rho+m}$, is insignificant, one can approximate the first-order condition (53) as follows:

$$
\frac{r}{\rho+m}=\underbrace{\frac{f-m}{\rho+m}}_{\approx 0}+\frac{1}{\varpi} \approx \frac{1}{\varpi} \Leftrightarrow \frac{\rho+m}{r} \approx \varpi .
$$

Plugging (51), (53) and (54) into the household's response (34) yields the optimal tax

$$
\begin{aligned}
\tau & =\frac{r-f-\rho}{r}=\frac{1}{r}\left(\frac{\rho+m}{\varpi}-m-\rho\right)=\frac{\rho+m}{r}\left(\frac{1}{\varpi}-1\right)=\varpi\left(\frac{1}{\varpi}-1\right) \\
& = \begin{cases}\left.\varpi\right|_{q=0}\left(\frac{1}{\left.\varpi\right|_{q=0}}-1\right)=\frac{\pi^{*} D^{*}}{1+\pi^{*} D^{*}} & \text { for } q=0, \\
0 & \text { for } q=1 .\end{cases}
\end{aligned}
$$

This result can be rephrased by the following proposition:

Proposition 3. Before the disaster, the optimal tax on capital income is an increasing function of the expected relative damage $\pi^{*} D^{*}$ as follows:

$$
\left.\tau\right|_{q=0} \approx \frac{\pi^{*} D^{*}}{1+\pi^{*} D^{*}}
$$

After the disaster, that tax can be eschewed, $\left.\tau\right|_{q=1}=0$.

\subsection{Taxing health care}

Because optimal public policy leads to the Pareto optimum, where consumption per head, $c$, the fertility rate $f$ and the mortality rate $m$ are equal in the household's and the government's problems, then, by (26), (32) and (41), the ratio of the household's and the government's value functions, (33) and (47), is $\Upsilon / \Lambda=\Psi / \Phi=\varpi / \vartheta=\varpi$. From this, (13), (15), (51) and the comparison of the household's and the government's first-order conditions, (29) and (46), it follows that

$$
0=(\rho+m) \frac{\partial \Upsilon}{\partial m}=\sigma c^{\sigma-1} f^{\alpha \sigma} \ell^{\sigma}\left(k+z^{\prime}\right)-\frac{\Upsilon}{\rho+m}
$$




$$
\begin{aligned}
& =\sigma c^{\sigma-1} f^{\alpha \sigma} \ell^{\sigma}\left(k+z^{\prime}\right)-\frac{\varpi \Lambda}{\rho+m} \\
& \Leftrightarrow k+z^{\prime}=\frac{1}{\sigma c^{\sigma-1} f^{\alpha \sigma} \ell^{\sigma}} \frac{\varpi \Lambda}{\rho+m}=\varpi\left[k+(1+b) z^{\prime}\right] \Leftrightarrow \\
& b=\left(\frac{1}{\varpi}-1\right)\left(\frac{k}{z^{\prime}}+1\right)=\left(\frac{1}{\varpi}-1\right)\left(k \chi^{\prime}+1\right) \\
& = \begin{cases}\left(k \chi^{\prime}+1\right) \pi^{*} D^{*}>0 & \text { for } q=0, \\
0 & \text { for } q=1 .\end{cases}
\end{aligned}
$$

Noting (15), the result (55) can be rephrased as follows:

Proposition 4. Before the disaster, the tax on health care should be in proportion $\left(k \chi^{\prime}+1\right)$ to the expected relative loss for the disaster $\pi^{*} D^{*}$,

$$
\left.b\right|_{q=0}=\left(k \chi^{\prime}+1\right) \pi^{*} D^{*}>0
$$

where $k$ is capital per head and $\chi^{\prime}$ the marginal efficiency of personal health care $h$ in decreasing the mortality rate $m[c f .(14)]$. After the disaster, that tax can be eschewed, $\left.b\right|_{q=1}=0$.

Because a single household ignores the effect of its health care $h$ on the other households' mortality rate $m$ through the increase of population $L$ and pollution $P$, its demand for health care exceeds the socially optimal level before the occurrence of the disaster. Thus, the demand for health care must be discouraged by the $\operatorname{tax} b$. The more efficiently personal health care decreases mortality (i.e., the greater $\chi^{\prime}$ ), or the more capital $k$ each surviving person needs, the higher the tax $b$ must be. If health care is very inefficient in decreasing mortality (i.e., $\chi^{\prime}$ is close enough to 0 ), then the tax is roughly equal to the expected relative loss for the disaster, $b \approx \pi^{*} D^{*}$.

\section{Conclusions}

This study examines the optimal management of a market economy where (i) households decide on saving, health care and the number of their children, (ii) the government controls their activity only by linear taxes, and (iii) 
population growth and capital accumulation generate pollution, increasing the risk of a lethal environmental disaster. In this situation, it turns out that a rational government should perform the following precautionary policy.

To implement Pareto optimality - i.e., to internalize the external link from population growth and capital accumulation to welfare though pollution and mortality - it is necessary to set precautionary taxes (i.e., taxes prior to the disaster) on capital income and the demand for health care. These are increasing functions of the expected relative damage of the disaster. The specific tax rules are given by Propositions 2, 3 and 4. In particular, Harford's (1997, 1998) parental tax is wholly unnecessary in this setup. In addition, only the revenue raising-poll tax is needed.

There are two reasons for this sharp result. First, because there is no incremental contribution of pollution to the mortality rate, then there is only the precautionary, but no maintenance motive for the government to intervene. Second, because the mortality rate can be decreased by spending on health care, then the mortality shock turns into an increase in the cost of health care, which has the same effect as an exogenous fall of income.

\section{Appendix: the approximation of the relative damage $D$}

Because, by (35) and (37), the fertility rate $f$ doesn't, but consumption per head, $c$, does depend on the shock $q$, the partial derivative of the value function (47) with respect to the shock $q$ is negative:

$$
\frac{\partial \Psi}{\partial q}(k, P, q, \zeta)=\frac{\partial}{\partial q} \max _{s, v, m} \frac{\varpi c^{\sigma} f^{\alpha \sigma} \ell^{\sigma}}{\rho+m}=\sigma \frac{\Psi}{c} \frac{\partial c}{\partial q}=-\frac{\sigma \delta}{c} \Psi(k, P, q, \zeta)<0 .
$$

Consider now what happens for the value function (47) if $q$ jumps discretely from 0 to 1. Applying the mean value theorem to (47), and noting (56), one obtains the following: there exists a value $\xi \in(0,1)$ so that

$$
\Psi(k, P, 1, \zeta)-\Psi(k, P, 0, \zeta)=\frac{\partial \Psi}{\partial q}(k, P, \xi, \zeta)=-\frac{\sigma \delta}{\left.c\right|_{q=\xi}} \Psi(k, P, \xi, \zeta)<0
$$

Furthermore, from (37) it follows that $\left.c\right|_{q=\xi}=\left.c\right|_{q=0}-\delta \xi$. Given this, (47) 
and (57), the relative damage (40) can be approximated by

$$
\begin{aligned}
& D(k, P, \zeta) \doteq \frac{\Psi(k, P, 0, \zeta)-\Psi(k, P, 1, \zeta)}{\Psi(k, P, 0, \zeta)}=\frac{\sigma \delta}{\left.c\right|_{q=\xi}} \frac{\Psi(k, P, \xi, \zeta)}{\Psi(k, P, 0, \zeta)} \\
& =\frac{\sigma \delta}{\left.c\right|_{q=\xi}}\left(\frac{\left.c\right|_{q=\xi}}{\left.c\right|_{q=0}}\right)^{\sigma}=\frac{\sigma \delta}{\left.c\right|_{q=0}}\left(\frac{\left.c\right|_{q=\xi}}{\left.c\right|_{q=0}}\right)^{\sigma-1}=\frac{\sigma \delta}{\left.c\right|_{q=0}}\left(1-\frac{\xi \delta}{\left.c\right|_{q=0}}\right)^{\sigma-1}>0 \\
& \text { with } \lim _{\frac{\delta}{\left.c\right|_{q=0}} \rightarrow 0}\left[\frac{\left.c\right|_{q=0}}{\sigma \delta} D(k, P, \zeta)\right]=\lim _{\frac{\delta}{\left.c\right|_{q=0}} \rightarrow 0}(1-\xi \underbrace{\frac{\delta}{\left.c\right|_{q=0}}}_{\rightarrow 0})^{\sigma-1}=1 \\
& \text { and } \lim _{\frac{\delta}{\left.c\right|_{q=0} \rightarrow 0}} D(k, P, \zeta)=\frac{\sigma \delta}{\left.c\right|_{q=0}} .
\end{aligned}
$$

Noting (19), the result (58) leads to the approximation $D(k, P, \zeta) \approx \sigma \frac{\delta}{c_{q=0}}$.

\section{Acknowledgments}

The author thanks the two anonymous reviewers and the editors for constructive comments and IIASA (Laxenburg, Austria) for hospitality during my visit in November 2018 when this paper was started.

\section{References:}

Becker, G.S., 1981. A treatise on the family. Cambridge (Mass.): Harvard University Press.

de Zeeuw, A., \& Zemel, A. (2012) Regime shifts and uncertainty in pollution control. Journal of Economic Dynamics and Control, 36, 939-950.

Dixit, A., \& Pindyck, K. (1994) Investment under uncertainty. Princeton: Princeton University Press.

Dockner, E.J., \& Long, N.V. (1993) International pollution control: cooperative versus noncooperative strategies. Journal of Environmental Economics and Management, 24, 13-29.

Harford, J.D. (1997) Stock pollution, child-bearing externalities, and the social discount rate. Journal of Environmental Economics and Management, 33, 94-105. 
Harford, J.D. (1998). The ultimate externality. The American Economic Review, 88, 260-265.

Haurie, A., \& Moresino, F. (2006) A stochastic control model of economic growth with environmental disaster prevention. Automatica, 42, 1417-1428.

Kamien, M.I., \& Schwartz, N. (1981) Dynamic optimization. The calculus of variations and optimal control in economics and management. New York: North-Holland.

Lehmijoki, U., \& Palokangas, T. (2018) Optimal population policy with deadly emissions. Paper presented in the $5^{\text {th }}$ International Workshop on Economic Growth, Environment and Natural Resources, 2019, European University at St. Petersburg, Russia.

Merton, R.C. (1971). Optimum consumption and portfolio rules in a continuous-time model. Journal of Economic Theory, 3, 373-413.

Palokangas, T. (2018) Optimal taxation with endogenous fertility and health-damaging emissions. IFAC PapersOnLine 51 (32), 79-83.

Polasky, A., de Zeeuw, A., \& Wagener, F. (2011) Optimal management with potential regime shifts. Journal of Environmental Economics and Management 62, 229-240.

Tsur, Y., \& Zemel, A. (2008) Regulating environmental threats. Environmental and Resource Economics, 39, 297-310.

Tsur, Y., \& Zemel, A. (2009) Endogenous discounting and climate policy. Environmental and Resource Economics, 44, 507-520.

Uzawa, H. (1968) Time preference, the consumption function, and optimum asset holdings. In: Wolfe J (ed) Value, Capital, and Growth. Aldine, Chicago (Chapter 21).

Van der Ploeg, F., \& de Zeeuw, A. (1992) International aspects of pollution control. Environmental and Resource Economics, 2, 117-139. 